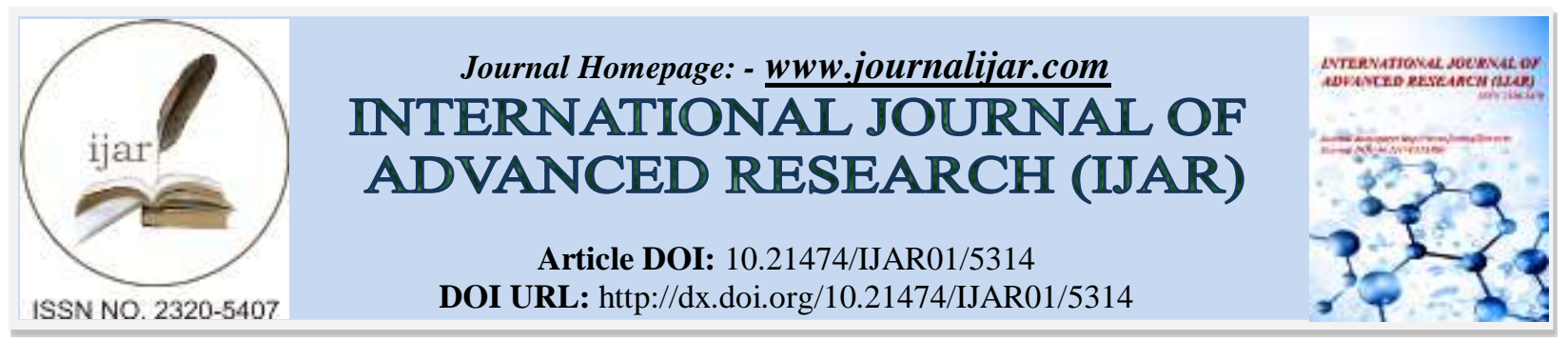

RESEARCH ARTICLE

\title{
BREAST CANCER KNOWLEDGE, ATTITUDES AND PRACTICE AMONG WOMEN IN KING KHALID ARMED FORCES HOSPITAL, TABUK 2017.
}

1. MBBS, Family Medicine Resident.

Nada Saleh Albalawi ${ }^{1}$ and Khalil Abu Jamilah ${ }^{2}$.

2. consultant of Family Medicine, King Khalid Armed Forces hospital, Tabuk.

\section{Manuscript Info}

Manuscript History

Received: 03 July 2017

Final Accepted: 05 August 2017

Published: September 2017

Key words:-

Breast Cancer, Knowledge, attitudes ,Practice.

\begin{abstract}
Objectives: This study aimed to assess knowledge, attitude and practice regarding breast cancer and its screening among women in King Khalid Armed Forces Hospital, Tabuk 2017.

Methods: It was cross sectional study-facility based study carried out in Tabuk city including a random sample of women (336) aged 18 years and above attending family medicine clinic and women health center, King Khalid Armed Forces hospital throughout the period between $1^{\text {st }}$ of March and 30 of April, 2017. Breast cancer awareness measure (CAM) validated questionnaire was used to collect the data.

Results: The age of almost one-third of them $(34.2 \%)$ ranged between 30 and 39 years. Overall, the sufficient overall knowledge regarding breast cancer was observed among $13.1 \%$ of the participants. Multivariate logistic regression analysis revealed that older women were more likely to have insufficient knowledge about breast cancer compared to younger women $(\mathrm{AOR}=2.98,95 \% \mathrm{CI}=1.03-8.68, \mathrm{p}=0.016$ and $\mathrm{AOR}=2.83,95 \% \mathrm{CI}=0.05-14.79, \mathrm{p}=0.049$, respectively). Most of women $(75.6 \%)$ rarely or never checked their breast , $8 \%$ did this at least once a month, $76.5 \%$ were not at all confident in noticing breast changes whereas only $1.2 \%$ of them were fairly confident, $27.4 \%$ saw a doctor about noticed breast changed whereas $44.9 \%$ of the women never noticed breast changes.

Conclusion: Minority of adult women attending family medicine clinic and women health center, King Khalid Armed Forces hospital, Tabuk have sufficient knowledge of breast cancer. Most of them rarely or never checked their breast and not at all confident in noticing breast changes.
\end{abstract}

Copy Right, IJAR, 2017,. All rights reserved.

\section{Introduction:-}

Breast Cancer epidemiology :-

According to the world health organization (WHO) :

Breast cancer is the top cancer in women worldwide. ${ }^{[1]}$ 


\section{According to CENTER FOR DISEASE CONTROL AND PREVENTION (CDC ):-}

Worldwide in 2012 (the latest available year for information), breast cancer was the second most common cancer ( $12 \%$ of all cancers diagnosed; 1.7 million people). Death from breast cancer was (6\% of all cancer deaths; 522,000 people). ${ }^{[2]}$

\section{Incidence of breast cancer in Saudi Arabia:-}

-Based on the last cancer incidence report of Saudi cancer registry 2013 that published on June 2016. The breast cancer was the most common cancer among Saudi population in 2013 for all ages, the number of cases was 1874 case in percentage of $16.1 \%$. Breast cancer was the most common cancer in Saudi women in percentage of $29.1 \%$.In Tabuk city, the breast cancer was the most common cancer among women in percentage of $24.9 \%$. The median age at the diagnosis of breast cancer among Women in Saudi Arabia 2013 was 50 year, ranged between 20 and 116 years. ${ }^{[3]}$

\section{Factors associated with increased risk of breast cancer:-}

The Age is strongly related to the breast cancer risk, Family history and genetics factor, Reproductive age as late age at first giving birth, early age at menarche, late age at menopause, Using of hormonal contraceptive , Using of hormone replacement therapy, Overweight and obesity, Height (Greater adult attained height is cause of postmenopausal breast cancer but in pre-menopausal women, probably the height is cause of breast cancer), More breast density, Benign breast disease, Atypical hyperplasia of breast, Previous history of breast cancer, Isonizing radiation exposure, Dietary fat and alcohol consumption, Tobacco smoking, Medical disease (diabetes mellitus , High bone mineral density and Autoimmune thyroiditis) ${ }^{[4]}$

\section{Factors associated with decrease risk of breast cancer:-}

Multiparty, breast feeding, physical activity, also, some diet is associated with decreasing the risk of breast cancer: vegetable and fruit, dietary fibers. ${ }^{[4]}$

\section{Symptoms of breast cancer:-}

Change in the size, shape of the breast, Lump in a breast or axilla, Skin changes as (skin rash ,puckering, dimpling), Abnormal Discharge from a nipple, Changes in the position of a nipple, Pain in the breast. ${ }^{[5]}$

\section{Screening by mammogram:-}

Clinical Practice Guideline on the Use of Screening Strategies for the Detection of Breast Cancer in Saudi Arabia:

"The Ministry of Health of Saudi Arabia guideline panel suggests screening with mammography in women aged 50-69 years every 2 years Conditional recommendation; moderate-quality evidence) ". [6]

"The USPSTF recommends biennial screening mammography for women aged 50 to 74 years". . ${ }^{[7]}$

\section{Breast Self-Examination and clinical breast examination:-}

1. "The Ministry of Health of Saudi Arabia guideline panel suggests that self-breast examination not be used as a single method of screening for breast cancer in women of all ages. (Conditional recommendation; very- low quality evidence ". ${ }^{[6]}$

2. "The Ministry of Health of Saudi Arabia guideline panel suggests that clinical breast examination by a health care professional not be used as a single method of screening for breast cancer in women of all ages. (Conditional recommendation; no evidence)“. [6]

\section{Rational :}

1. No data available about the level of awareness of our patients in Tabuk city about breast cancer .

2. Measuring of breast cancer related knowledge and practices is the key to direct the breast cancer awareness campaigns for women that conducted in many health and non-health centers.

3. Increasing the awareness of breast cancer among women has long term impact on early patient presentation, as has been suggested by studies in the United Kingdom. ${ }^{[8]}$

4. Breast cancer awareness is significantly important in studies done in low income countries found that the awareness of breast self-examination had significant impact on early presentation. ${ }^{[9]}$

\section{Problem Statements:-}

1. Breast cancer is common in developing countries where the majority of cases are diagnosed in late stages. ${ }^{[1]}$ 
2. Diagnosis of breast cancer at advanced stages contributes to the high mortality rate among women due to breast cancer, which can be attributed to the low levels of awareness. ${ }^{[10]}$

3. low levels of cancer awareness have been found to be an important risk factor for delay in presentation of cancer by the patients. ${ }^{[1]]}$

This study is aimed to assess the knowledge and attitude about breast cancer and to identify the practice about the breast self-examination among women in King Khalid Armed Forces Hospital, Tabuk 2017.

\section{Material and Methods:-}

It was a cross sectional study-facility based study, carried out among all women aged 18 years and above attending family medicine clinic and women health center at King Khalid Armed Forces hospital, Tabuk city, northwestern Saudi Arabia. Based on published study conducted in Riyadh, the prevalence of breast cancer awareness was $30 \%$.

${ }^{[12]}$ Sample size was estimated to be 336 and was increased to 360 subjects to compensate for the potential nonresponse.

\section{Study variables:-}

Age, marital status, level of education, nationality, income, risk factors of breast cancer, signs of breast cancer, information and behavior about breast examination and breast cancer screening.

\section{Study tool:-}

Breast cancer awareness measure (CAM) validated questionnaire was used to collect the data. Modified Arabic version of breast cancer awareness measure (CAM) was used. The Breast-CAM was developed by Cancer Research UK, King's College London and University College London in 2009 and validated with the support of Breast Cancer Care and Breakthrough Breast Cancer. ${ }^{[13]}$

Collected Data were entered, stored and analyzed using the statistical package for Social Science (SPSS) programme, V22 for window. Chi-square test was used for testing the association among categorical variables. P value equal or less than 0.05 was considered as statistical significant.

For knowledge questions, each correct answer was assigned a score of "1" while incorrect or missing or don"t know answers were assigned a score of "0". Total score was computed for every participant and knowledge percentage was computed. Those who had below $50 \%$ of the total percentage score were considered as having "insufficient knowledge" whereas those scored $50 \%$ or more were considered as having "sufficient knowledge".

Approvals from administration in King Khalid Armed Forces hospital were obtained. Confidentiality was assured to all participants. Informed consent was obtained by every patient before starting the interview.

\section{Results:-}

\section{Socio-demographic characteristics:-}

The study included 336 women out of a targeted sample of 360 giving a response rate of $93.3 \%$. Their sociodemographic characteristics are summarized in table 1. The age of almost one-third of them (34.2\%) ranged between 30 and 39 years whereas that of $11.9 \%$ of them was 60 years or above. Majority of the participants were Saudis $(99.1 \%)$ and reside inside Tabuk city (90.8\%). Also majority of them $(86.8 \%)$ were married. About one-third of the women $(34.2 \%$ were illiterate whereas $16.1 \%$ were university graduated. Regarding family income per month, 47.3\% of them had more than 10000 whereas $11.6 \%$ had less than 5000 Saudi Riyals. (Table 1).

\section{Knowledge about breast cancer:- Symptoms of breast cancer:-}

From table 2, it is seen that majority of the women (92.9\%) could recognize presence of a lump or thickness in breast as a symptom of breast cancer. Approaching half of the participants (47.3\%) knew correctly that pain in one breast could be a symptom of breast cancer. Almost one-quarter of them could recognize that presence of a lump or thickness under armpit (27.7\%), changes in the size of breast or nipple (27.7\%) and discharge or bleeding from nipple (25.6\%) as symptoms of breast cancer. Other symptoms of breast cancer such as change in the nipple position, pulling of nipple, puckering or dimpling of breast skin, nipple rash, redness of breast skin and changes in the shape of breast or nipple were recognized by minority of the participants (12.5\% to 14\%). (Table 2). 
The sufficient knowledge regarding symptoms of breast cancer was observed among $13.4 \%$ of the participants . ( Figure 1).

\section{Risk factors for breast cancer:-}

More than half of the participants $(54.5 \%)$ could recognize past history of breast cancer as a risk factor. More than one third of women $(36.3 \%)$ knew that using hormonal replacement therapy is a risk factor for breast cancer. More than one quarter of them $(27.7 \%)$ knew correctly that having a close relative with breast cancer is a risk factor for breast cancer. Minority of the participants could recognize that being overweight, having children later on in life or not at all, starting periods at an early age, having a late menopause, physical inactivity and aged of 50 years are risk factors for breast cancer. (Table 3)

The sufficient knowledge regarding risk factors for breast cancer was observed among only $8.6 \%$ of the participants. (Figure 2).

\section{Screening for breast cancer:-}

As demonstrated in table 4, minority of the women could recognize that the age at which women first invited to breast cancer screening programme according to guidelines of Saudi Ministry of Health is 50 years (1.8\%) and the age at which women receive last invitation to breast cancer screening programme according to guidelines of Saudi Ministry of Health is 69 years $(0.3 \%)$. Only one woman reported invitation for breast cancer screening $(0.3 \%)$ and she was the only one who had breast cancer screening $(0.3 \%)$.

The sufficient knowledge regarding screening for breast cancer was observed among only $2.1 \%$ of the participants . (Figure 3).

Factors associated with overall knowledge regarding breast cancer:-

Overall, the sufficient overall knowledge regarding breast cancer was observed among $13.1 \%$ of the participants . (Figure 4).

From table 5, it is shown that $27.7 \%$ of women aged between 18 and 29 years compared to $6.2 \%$ of women aged between 50 and 59 years and $2.5 \%$ of those aged 60 years or more had sufficient knowledge about breast cancer. These differences were statistically significant, $\mathrm{p}=0.001$. Single women had higher sufficient knowledge about breast cancer compared to married, divorced and widowed women ( $27.3 \%$ versus $13 \%$ and zero\%, respectively), $\mathrm{p}=0.027$. University graduated women were more knowledgeable about breast cancer compared to illiterate women (27.8\% versus $4.3 \%), \mathrm{p}=0.001$. Women`s nationality, residence location and income were not significantly associated with knowledge about breast cancer. (Table 4) .

Multivariate logistic regression analysis revealed that women in the age groups 30-39 and $\geq 60$ years were more likely to have insufficient knowledge about breast cancer compared to those aged 18-29 years $(\mathrm{AOR}=2.98,95 \%$ $\mathrm{CI}=1.03-8.68, \mathrm{p}=0.016$ and $\mathrm{AOR}=2.83,95 \% \mathrm{CI}=0.05-14.79, \mathrm{p}=0.049$, respectively). Compared to illiterate women, high school and university graduated women were at lower significant risk for having insufficient knowledge about breast cancer $(\mathrm{AOR}=0.07,95 \% \mathrm{CI}=0.01-0.50, \mathrm{p}=0.009$ and $\mathrm{AOR}=0.03,95 \% \mathrm{CI}=0.001-0.27$, $\mathrm{p}=0.001$, respectively $)$. (Table 5) .

\section{Confidence, skills and behaviors in relation to breast changes:-}

The results show that $75.6 \%$ of the participants rarely or never checked their breast and only $8 \%$ did this at least once a month. Also, $76.5 \%$ were not at all confident in noticing breast changes whereas only $1.2 \%$ of the participants were fairly confident. Almost one-quarter of them (27.4\%) saw a doctor about noticed breast changes whereas another $27.4 \%$ did not do this and $44.9 \%$ of the women never noticed breast changes.

\section{Factors associated with frequency of checking breast cancer:-}

Table 6 shows that $15.2 \%$ of women in the age group 18-29 years compared to none of those aged 50 years and more checked their breasts at least once/month. This difference was statistically significant, $\mathrm{p}<0.001$. Almost a quarter $(24.1 \%)$ of university graduated women compared to none of illiterate women checked their breasts at least once/month. This difference was statistically significant, $\mathrm{p}<0.001$. Women with higher family income $(>10000$ $\mathrm{SR} / \mathrm{month}$ ) were more likely to check their breasts at least once/month compared to those whose income was less 
than $5000 \mathrm{SR} /$ month $(10.7 \%$ versus $0 \%), \mathrm{p}=0.011$. Women`s nationality, residence location and marital status were not significantly associated with frequency of checking breasts. (Table 6) .

Factors associated with women`s confidence in noticing breast change:-

Table 7 shows that $28.7 \%$ of women in the age group 30-39 years compared to none of those aged 50 years and more were slightly or fairly confident in noticing their breast changes. This difference was statistically significant, $\mathrm{p}<0.001$. More than forty percent $(40.7 \%)$ of university graduated women compared to none of illiterate women were slightly or fairly confident in noticing their breast changes. This difference was statistically significant, $\mathrm{p}<0.001$. Women`s nationality, residence location, marital status and income were not significantly associated with confidence in noticing breast changes. (Table 7).

Factors associated with seeing doctors about noticed breast change:-

Table 8 shows that $38.5 \%$ of women in the age group 50-59 years compared to 12.8 of those aged between 18 and 29 years saw doctors for noticed breast change. This difference was statistically significant, $\mathrm{p}<0.001$. More than One third $(38.1 \%)$ of divorced/widowed women compared to $9.1 \%$ of single women saw doctors for noticed breast change. This difference was statistically significant, $\mathrm{p}=0.004$. More than half $(53.7 \%)$ of university graduated women compared to $21.9 \%$ of illiterate women never noticed breast changes. This difference was statistically significant, $\mathrm{p}<0.001$. Exactly half of women whose income ranged between 5000 and 10000 compared to $18.4 \%$ of those whose income was less than $5000 \mathrm{SR} /$ month never noticed their breast changes. This difference was statistically significant, $\mathrm{p}=0.003$. Less than half of women reside in Tabuk city (46.6\%) compared to $30 \%$ of those reside in Tabuk rural area never noticed their breast changes. This difference was statistically significant, $\mathrm{p}=0.043$. Women`s nationality was not significantly associated with seeing doctor about noticed breast changes. (Table 8 ).

Table 1:- Socio-Demographic Characteristics Of The Participants.

\begin{tabular}{|l|l|l|}
\hline & Frequency $(\mathrm{n}=336)$ & Percentage \\
\hline Age in years & & \\
$18-29$ & $\mathbf{4 7}$ & $\mathbf{1 4 . 1}$ \\
$30-39$ & $\mathbf{1 1 5}$ & $\mathbf{3 4 . 2}$ \\
$40-49$ & $\mathbf{6 9}$ & $\mathbf{2 0 . 5}$ \\
$50-59$ & $\mathbf{6 5}$ & $\mathbf{1 9 . 3}$ \\
$\geq 60$ & $\mathbf{4 0}$ & $\mathbf{1 1 . 9}$ \\
\hline Nationality & $\mathbf{3 3 3}$ & \\
Saudi & $\mathbf{3}$ & $\mathbf{9 9 . 1}$ \\
Non-Saudi & & $\mathbf{0 . 9}$ \\
\hline Residency location & $\mathbf{3 0 5}$ & \\
Tabuk city & $\mathbf{3 1}$ & $\mathbf{9 0 . 8}$ \\
Rural area of Tabuk & & $\mathbf{9 . 2}$ \\
\hline Marital status & $\mathbf{2 2}$ & \\
Single & $\mathbf{2 9 2}$ & $\mathbf{6 . 6}$ \\
Married & $\mathbf{2 2}$ & $\mathbf{8 6 . 8}$ \\
Divorced/widowed & & $\mathbf{6 . 6}$ \\
\hline Educational level & $\mathbf{1 1 5}$ & \\
Illiterate & $\mathbf{4 1}$ & $\mathbf{3 4 . 2}$ \\
Primary school & $\mathbf{2 5}$ & $\mathbf{1 2 . 2}$ \\
Intermediate school & $\mathbf{1 0 1}$ & $\mathbf{7 . 4}$ \\
High school & $\mathbf{5 4}$ & $\mathbf{3 0 . 1}$ \\
University & $\mathbf{3 9}$ & $\mathbf{1 6 . 1}$ \\
\hline Income (SAR/month) & $\mathbf{1 3 8}$ & $\mathbf{1 1 . 6}$ \\
<5000 & $\mathbf{1 5 9}$ & $\mathbf{4 1 . 1}$ \\
5000-10000 & & $\mathbf{4 7 . 3}$ \\
\hline$>10000$ & &
\end{tabular}

Table 2:- Knowledge Of The Participants About Symptoms Of Breast Cancer. Breast cancer symptoms

\begin{tabular}{|l|l|}
\hline \multicolumn{2}{|l|}{ Right answer } \\
\hline No. & $\%$ \\
\hline
\end{tabular}




\begin{tabular}{|l|l|l|}
\hline Change in the nipple position & $\mathbf{4 5}$ & $\mathbf{1 3 . 4}$ \\
\hline Pulling of nipple & $\mathbf{4 6}$ & $\mathbf{1 3 . 7}$ \\
\hline Pain in one breast & $\mathbf{1 5 9}$ & $\mathbf{4 7 . 3}$ \\
\hline Puckering or dimpling of breast skin & $\mathbf{4 7}$ & $\mathbf{1 4 . 0}$ \\
\hline Discharge or bleeding from nipple & $\mathbf{8 6}$ & $\mathbf{2 5 . 6}$ \\
\hline Lump or thickness in breast & $\mathbf{3 1 2}$ & $\mathbf{9 2 . 9}$ \\
\hline Nipple rash & $\mathbf{4 2}$ & $\mathbf{1 2 . 5}$ \\
\hline Redness of breast skin & $\mathbf{4 4}$ & $\mathbf{1 3 . 1}$ \\
\hline Lump or thickness under armpit & $\mathbf{9 3}$ & $\mathbf{2 7 . 7}$ \\
\hline Changes in the size of breast or nippl & $\mathbf{9 3}$ & $\mathbf{2 7 . 7}$ \\
\hline Changes in the shape of breast or nipple & $\mathbf{4 7}$ & $\mathbf{1 4 . 0}$ \\
\hline
\end{tabular}

Table 3: Knowledge of the participants about Risk factors for breast cancer.

\begin{tabular}{|c|c|c|}
\hline \multirow[t]{2}{*}{ Risk factors for breast cancer } & \multicolumn{2}{|c|}{ Right answer } \\
\hline & No. & $\%$ \\
\hline Having a past history of breast cancer & 183 & 54.5 \\
\hline Using hormonal replacement therapy & 122 & 36.3 \\
\hline Being overweight (BMI over $25 \mathrm{Kg} / \mathrm{m}^{2}$ ) & 29 & 8.6 \\
\hline Having a close relative with breast cancer & 93 & 27.7 \\
\hline Having children later on in life or not at all & 29 & 8.6 \\
\hline Starting periods at an early age & 29 & 8.6 \\
\hline Having a late menopause & 29 & 8.6 \\
\hline Doing less than 30 minutes of moderate physical activity 5 minutes a week & 27 & 8.0 \\
\hline Aged 50 years & 12 & 3.6 \\
\hline
\end{tabular}

Table 4:- Factors associated with overall knowledge regarding breast cancer

\begin{tabular}{|c|c|c|c|}
\hline & \multicolumn{2}{|c|}{ Breast cancer knowledge } & \multirow{2}{*}{$\begin{array}{l}\chi^{2} \\
\text { (p-value) }\end{array}$} \\
\hline & $\begin{array}{l}\text { Insufficient } \\
\mathrm{N}=292 \\
\mathrm{~N}(\%)\end{array}$ & $\begin{array}{l}\text { Sufficient } \\
N=44 \\
N(\%)\end{array}$ & \\
\hline $\begin{array}{l}\text { Age in years } \\
18-29(\mathrm{n}=47) \\
30-39(\mathrm{n}=115) \\
40-49(\mathrm{n}=69) \\
50-59(\mathrm{n}=65) \\
\geq 60(\mathrm{n}=40)\end{array}$ & $\begin{array}{l}34(72.3) \\
102(88.7) \\
56(81.2) \\
61(93.8) \\
39(97.5) \\
\end{array}$ & $\begin{array}{l}13(27.7) \\
13(11.3) \\
13(18.8) \\
4(6.2) \\
1(2.5) \\
\end{array}$ & $\begin{array}{l}17.78 \\
(0.001) \\
\end{array}$ \\
\hline $\begin{array}{l}\text { Nationality } \\
\text { Saudi }(n=333) \\
\text { Non-Saudi }(n=3)\end{array}$ & $\begin{array}{l}290(87.1) \\
2(66.7)\end{array}$ & $\begin{array}{l}43(12.9) \\
1(33.3)\end{array}$ & $0.345^{*}$ \\
\hline $\begin{array}{l}\text { Residency location } \\
\text { Tabuk city }(\mathrm{n}=305) \\
\text { Rural area of Tabuk }(\mathrm{n}=31)\end{array}$ & $\begin{array}{l}264(86.6) \\
28(90.3)\end{array}$ & $\begin{array}{l}41(13.4) \\
3(9.7)\end{array}$ & $0.398 *$ \\
\hline $\begin{array}{l}\text { Marital status } \\
\text { Single }(n=22) \\
\text { Married }(n=292) \\
\text { Divorced/widowed }(n=22)\end{array}$ & $\begin{array}{l}16(72.7) \\
254(87.0) \\
22(100 \\
\end{array}$ & $\begin{array}{l}6(27.3) \\
38(13.0) \\
0(0.0)\end{array}$ & $\begin{array}{l}7.20 \\
(0.027) \\
\end{array}$ \\
\hline $\begin{array}{l}\text { Educational level } \\
\text { Illiterate }(\mathrm{n}=115) \\
\text { Primary school }(\mathrm{n}=41) \\
\text { Intermediate school }(\mathrm{n}=25) \\
\text { High school }(\mathrm{n}=101) \\
\text { University }(\mathrm{n}=54)\end{array}$ & $\begin{array}{l}110(95.7) \\
36(87.8) \\
22(88.0) \\
85(84.2) \\
39(72.2) \\
\end{array}$ & $\begin{array}{l}5(4.3) \\
5(12.2) \\
3(12.0) \\
16(15.8) \\
15(27.8) \\
\end{array}$ & $\begin{array}{l}18.69 \\
(0.001)\end{array}$ \\
\hline $\begin{array}{l}\text { Income (SAR/month) } \\
<5000(\mathrm{n}=39)\end{array}$ & 38 (97.4) & $1(2.6)$ & \\
\hline
\end{tabular}




\begin{tabular}{|l|l|l|l|}
\hline $5000-10000(\mathrm{n}=138)$ & $120(87.0)$ & $18(13.0)$ & 4.77 \\
$>10000(\mathrm{n}=159)$ & $134(84.3)$ & $25(15.7)$ & $(0.092$ \\
\hline
\end{tabular}

* Fischer exact test

Table 5:- Socio-demographic predictors for insufficient breast cancer knowledge: Multivariate logistic regression analysis

\begin{tabular}{|c|c|c|c|}
\hline & $\begin{array}{lll}\begin{array}{l}\text { Adjusted } \\
\text { (AOR) }\end{array} & \text { Odds } & \text { ratio } \\
\end{array}$ & $\begin{array}{l}95 \% \text { Confidence } \\
\text { interval (CI) }\end{array}$ & p-value \\
\hline $\begin{array}{l}\text { Age in years } \\
18-29(\mathrm{n}=47)^{(\mathrm{a})} \\
30-39(\mathrm{n}=115) \\
40-49(\mathrm{n}=69) \\
50-59(\mathrm{n}=65) \\
\geq 60(\mathrm{n}=40)\end{array}$ & $\begin{array}{l}--- \\
2.98 \\
1.09 \\
1.03 \\
2.83\end{array}$ & $\begin{array}{l}--- \\
1.03-8.68 \\
0.12-1.68 \\
0.04-3.09 \\
0.05-14.79\end{array}$ & $\begin{array}{l}0.016 \\
0.120 \\
0.361 \\
0.049\end{array}$ \\
\hline $\begin{array}{l}\text { Educational level } \\
\text { Illiterate }(\mathrm{n}=115)^{(\mathrm{a})} \\
\text { Primary school }(\mathrm{n}=41) \\
\text { Intermediate } \mathrm{school}(\mathrm{n}=25) \\
\text { High school }(\mathrm{n}=101) \\
\text { University }(\mathrm{n}=54)\end{array}$ & $\begin{array}{l}--- \\
0.37 \\
0.17 \\
0.07 \\
0.03\end{array}$ & $\begin{array}{l}--- \\
0.06-2.44 \\
0.02-1.47 \\
0.01-0.50 \\
0.001-0.27\end{array}$ & $\begin{array}{l}0.304 \\
0.108 \\
0.009 \\
0.001\end{array}$ \\
\hline
\end{tabular}

${ }^{(a)}$ : Reference category

Variables of marital status and income were removed from the final model (not significant)

Table 6:- Factors associated with frequency of checking breast cancer among the participants

\begin{tabular}{|c|c|c|c|c|}
\hline & \multicolumn{3}{|c|}{ Frequency of checking the breast } & \multirow{2}{*}{$\begin{array}{l}\chi^{2} \\
(\mathrm{p} \text {-value })\end{array}$} \\
\hline & $\begin{array}{l}\text { Rarely/Never } \\
\mathrm{N}=254 \\
\mathrm{~N}(\%)\end{array}$ & \begin{tabular}{|l|} 
At least once/6 \\
months \\
$\mathbf{N}=52$ \\
$\mathbf{N}(\%)$
\end{tabular} & $\begin{array}{l}\text { At least } \\
\text { once/month } \\
\mathbf{N}=27 \\
\mathrm{~N}(\%)\end{array}$ & \\
\hline $\begin{array}{l}\text { Age in years } \\
18-29(\mathrm{n}=46) \\
30-39(\mathrm{n}=115) \\
40-49(\mathrm{n}=69) \\
50-59(\mathrm{n}=65) \\
\geq 60(\mathrm{n}=38)\end{array}$ & $\begin{array}{l}34(73.9) \\
63(54.8) \\
55(79.7) \\
65(100) \\
37(97.4)\end{array}$ & $\begin{array}{l}5(10.9) \\
36(31.3) \\
10(14.5) \\
0(0.0) \\
1(2.6)\end{array}$ & $\begin{array}{l}7(15.2) \\
16(13.9) \\
4(5.8) \\
0(0.0) \\
0(0.0)\end{array}$ & $\begin{array}{l}63.67 \\
(<0.001)\end{array}$ \\
\hline $\begin{array}{l}\text { Nationality } \\
\text { Saudi }(n=330) \\
\text { Non-Saudi }(n=3)\end{array}$ & $\begin{array}{l}252(76.4) \\
2(66.7)\end{array}$ & $\begin{array}{l}51(15.5) \\
1(33.3)\end{array}$ & $\begin{array}{l}27(8.2) \\
0(0.0)\end{array}$ & $\begin{array}{l}0.89 \\
(0.641)\end{array}$ \\
\hline $\begin{array}{l}\text { Residency location } \\
\text { Tabuk city }(n=305) \\
\text { Rural area of Tabuk }(n=28)\end{array}$ & $\begin{array}{l}229(75.1) \\
25(89.3)\end{array}$ & $\begin{array}{l}50(16.4) \\
2(7.1)\end{array}$ & $\begin{array}{l}26(8.5) \\
1(3.6)\end{array}$ & $\begin{array}{l}2.86 \\
(0.239)\end{array}$ \\
\hline $\begin{array}{l}\text { Marital status } \\
\text { Single }(\mathrm{n}=22) \\
\text { Married }(\mathrm{n}=290) \\
\text { Divorced/widowed }(\mathrm{n}=21)\end{array}$ & $\begin{array}{l}16(72.7) \\
219(75.5) \\
19(90.5)\end{array}$ & $\begin{array}{l}2(9.1) \\
49(16.9) \\
1(4.8)\end{array}$ & $\begin{array}{l}4(18.2) \\
22(7.6) \\
1(4.8)\end{array}$ & $\begin{array}{l}6.24 \\
(0.182) \\
\end{array}$ \\
\hline $\begin{array}{l}\text { Educational level } \\
\text { Illiterate }(\mathrm{n}=113) \\
\text { Primary school }(\mathrm{n}=41) \\
\text { Intermediate school }(\mathrm{n}=24) \\
\text { High school }(\mathrm{n}=101) \\
\text { University }(\mathrm{n}=54)\end{array}$ & $\begin{array}{l}111(98.2) \\
35(85.4) \\
22(91.7) \\
60(59.4) \\
26(48.1)\end{array}$ & $\begin{array}{l}2(1.8) \\
4(9.8) \\
1(4.2) \\
30(29.7) \\
15(27.8)\end{array}$ & $\begin{array}{l}0(0.0) \\
2(4.9) \\
1(4.2) \\
11(10.9) \\
13(24.1)\end{array}$ & $\begin{array}{l}80.53 \\
(<0.001)\end{array}$ \\
\hline $\begin{array}{l}\text { Income (SAR/month) } \\
<5000(\mathrm{n}=37) \\
5000-10000(\mathrm{n}=137)\end{array}$ & $\begin{array}{l}36(97.3) \\
107(78.1)\end{array}$ & $\begin{array}{l}1(2.7) \\
20(14.6)\end{array}$ & $\begin{array}{l}0(0.0) \\
10(7.3)\end{array}$ & 13.07 \\
\hline
\end{tabular}




\begin{tabular}{|l|l|l|l|l|}
\hline$>10000(\mathrm{n}=159)$ & $111(69.8)$ & $31(19.5)$ & $17(10.7)$ & $(0.011)$ \\
\hline
\end{tabular}

Table 7:- Factors associated with participants` confidence in noticing breast changes

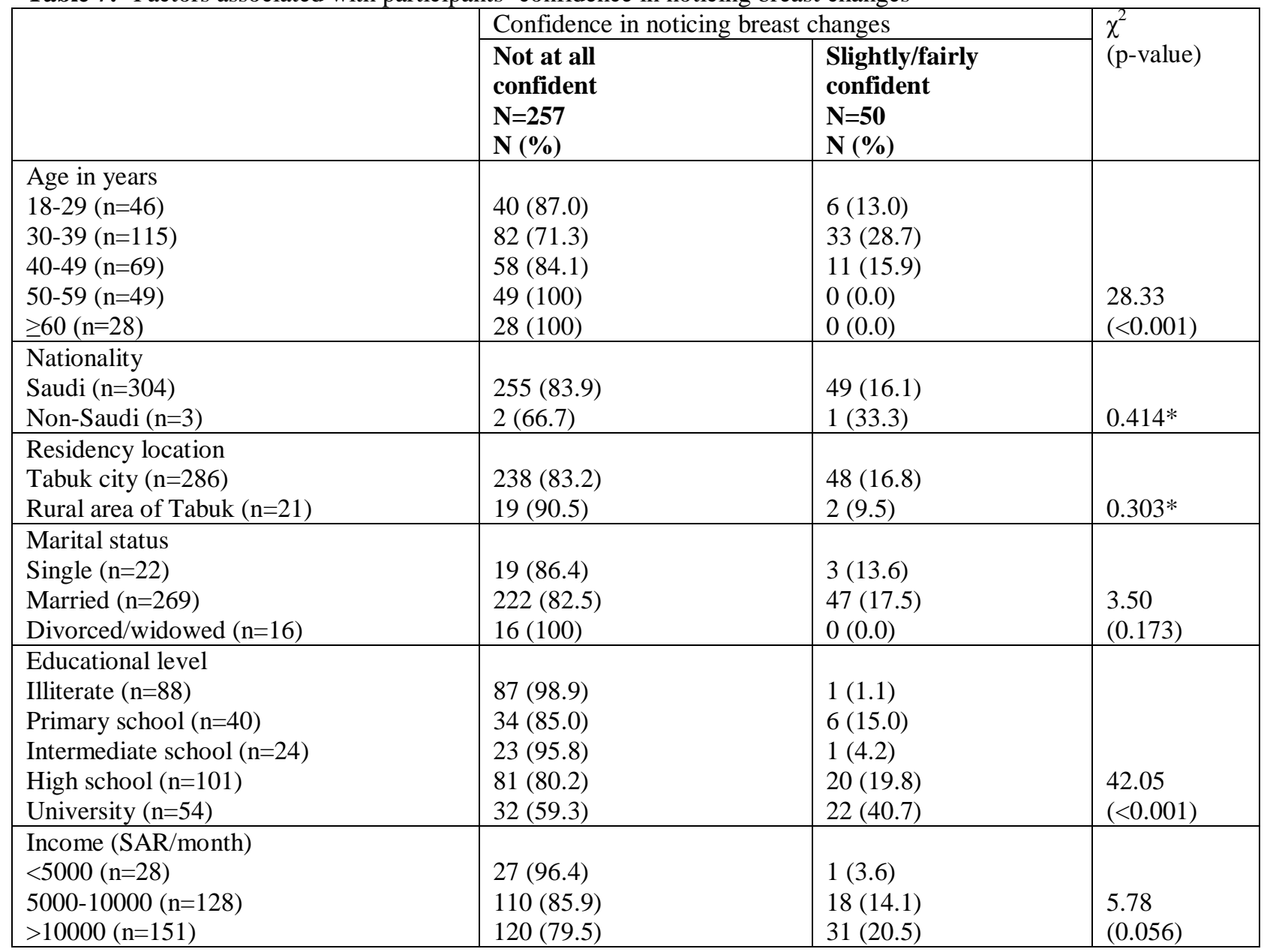

* Fischer exact test

Table 8:- Factors associated with seeing doctors about noticed breast changes

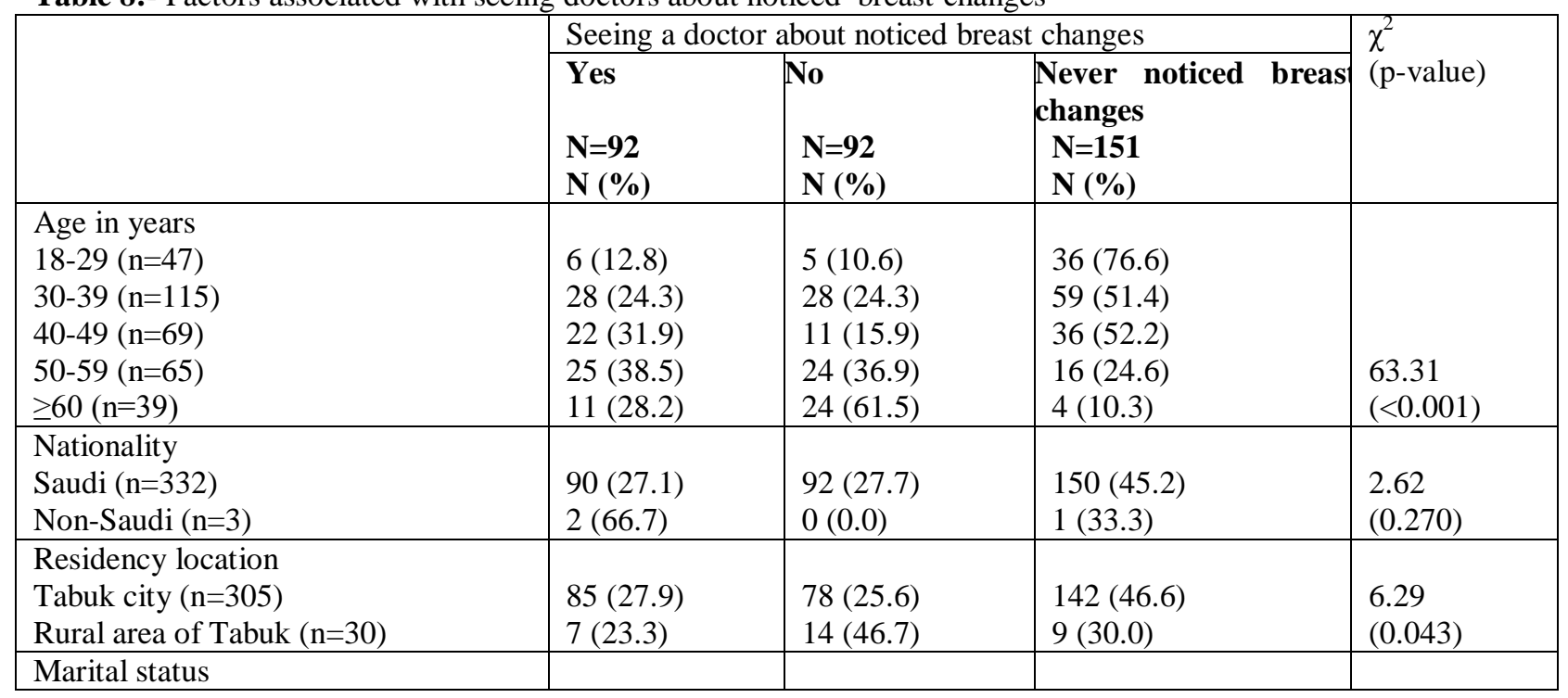




\begin{tabular}{|l|l|l|l|l|}
\hline Single $(\mathrm{n}=22)$ & $2(9.1)$ & $3(13.6)$ & $17(77.3)$ & \\
Married (n=292) & $82(28.1)$ & $80(27.4)$ & $130(44.5)$ & 15.19 \\
Divorced/widowed (n=21) & $8(38.1)$ & $9(42.9)$ & $4(19.0)$ & $(0.004)$ \\
\hline Educational level & & & & \\
Illiterate (n=114) & $39(34.2)$ & $50(43.9)$ & $25(21.9)$ & \\
Primary school (n=41) & $14(34.1)$ & $6(14.6)$ & $21(51.2)$ & \\
Intermediate school (n=25) & $5(20.0)$ & $4(16.0)$ & $16(64.0)$ & 47.37 \\
High school (n=101) & & & & \\
University (n=54) & $17(16.8)$ & $24(23.8)$ & $60(59.4)$ & \\
& $17(31.5)$ & $8(14.8)$ & $29(53.7)$ & $16.001)$ \\
\hline Income (SAR/month) & & & & \\
$<5000(\mathrm{n}=38)$ & $12(31.6)$ & $19(50.0)$ & $7(18.4)$ & $(0.003)$ \\
$5000-10000(\mathrm{n}=138)$ & $33(23.9)$ & $36(26.1)$ & $69(50.0)$ & $75(47.2)$ \\
$>10000(\mathrm{n}=159)$ & $47(29.6)$ & $37(23.3)$ & & \\
\hline
\end{tabular}

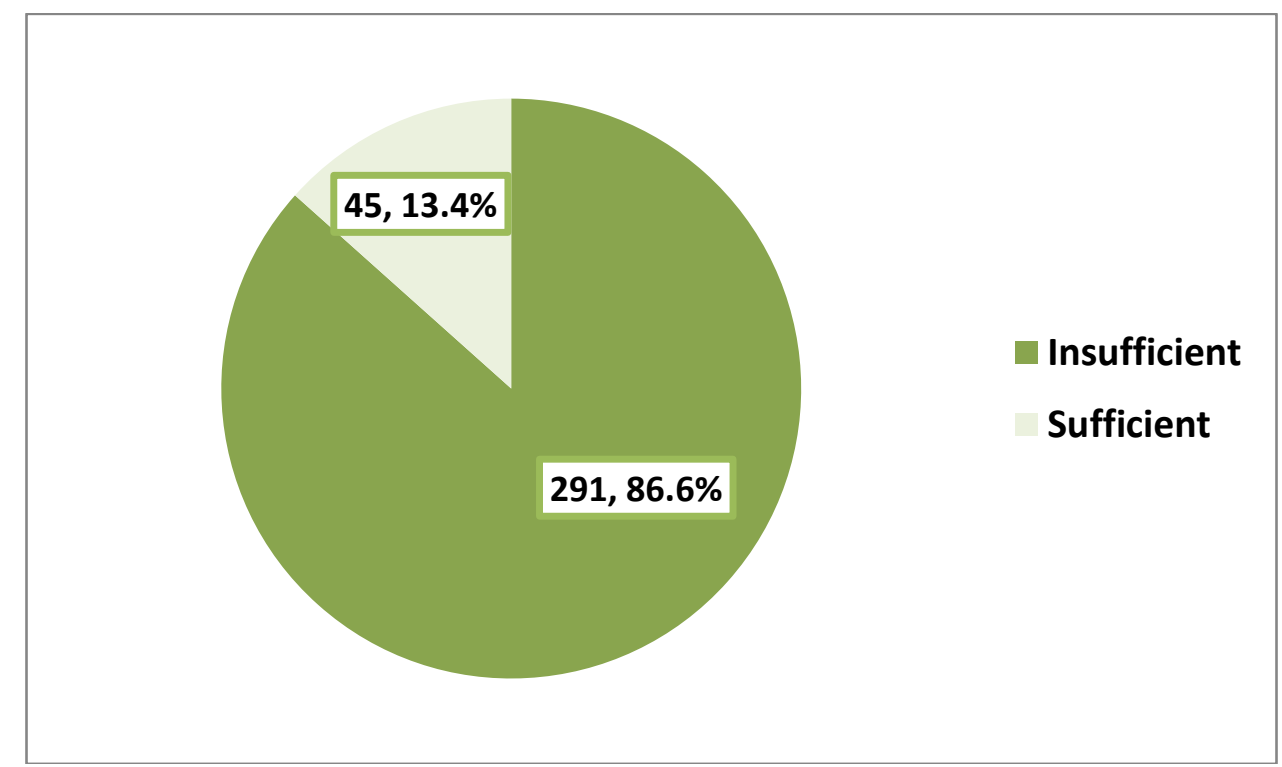

Figure 1:- Knowledge level of the participants regarding symptoms of breast cancer.

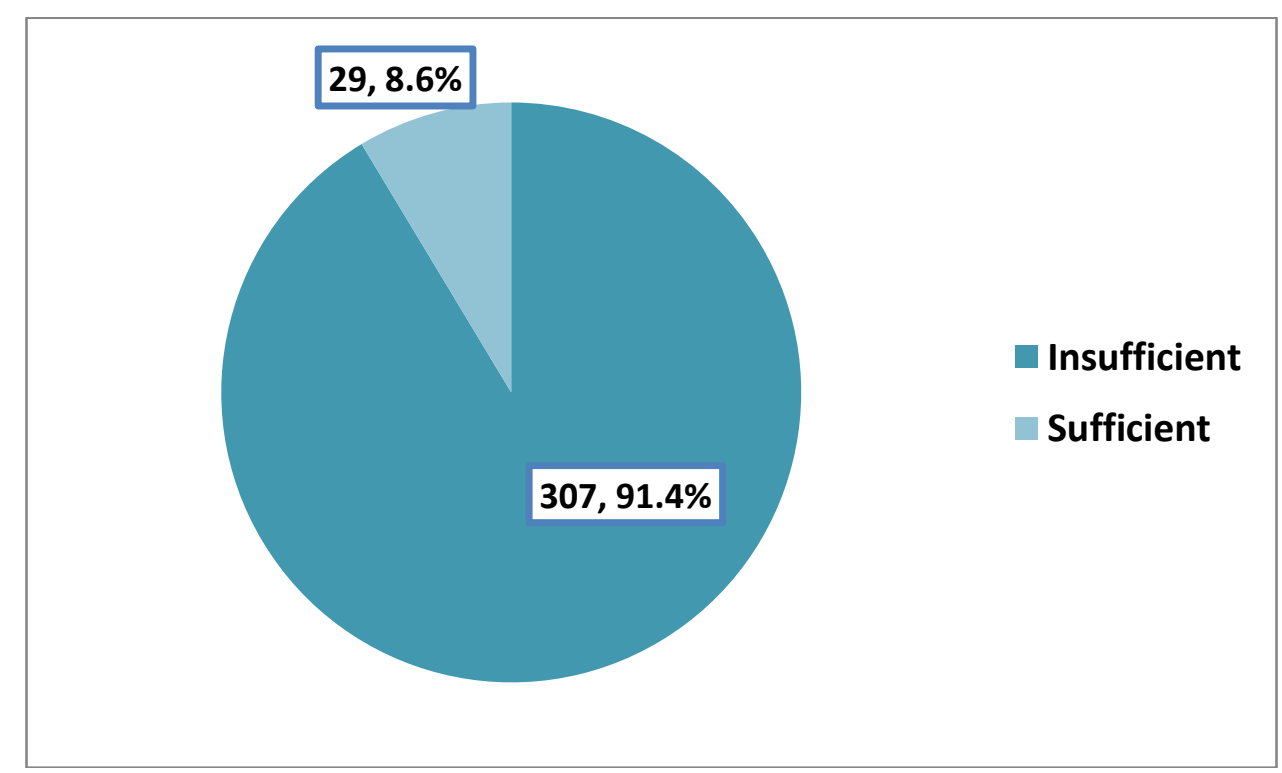

Figure 2:- Knowledge level of the participants regarding risk factors for breast cancer 


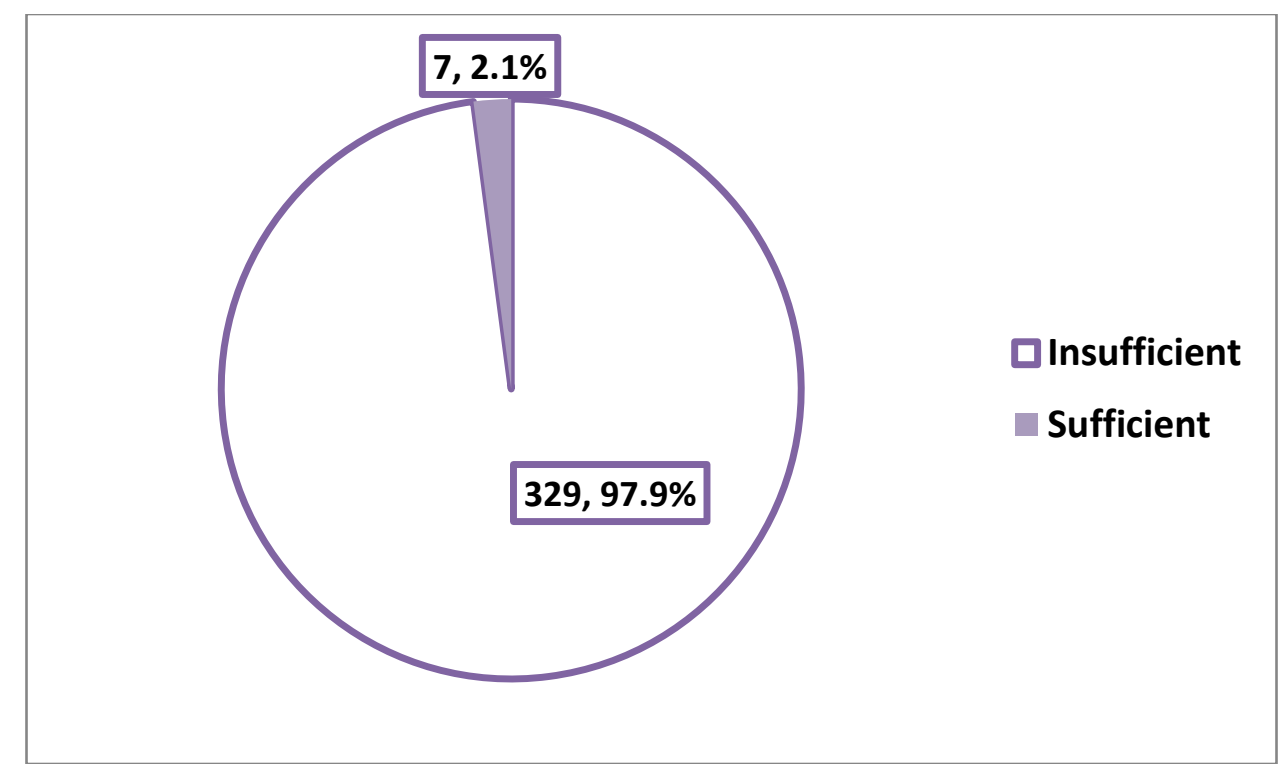

Figure 3:- Knowledge level of the participants regarding screening for breast cancer.

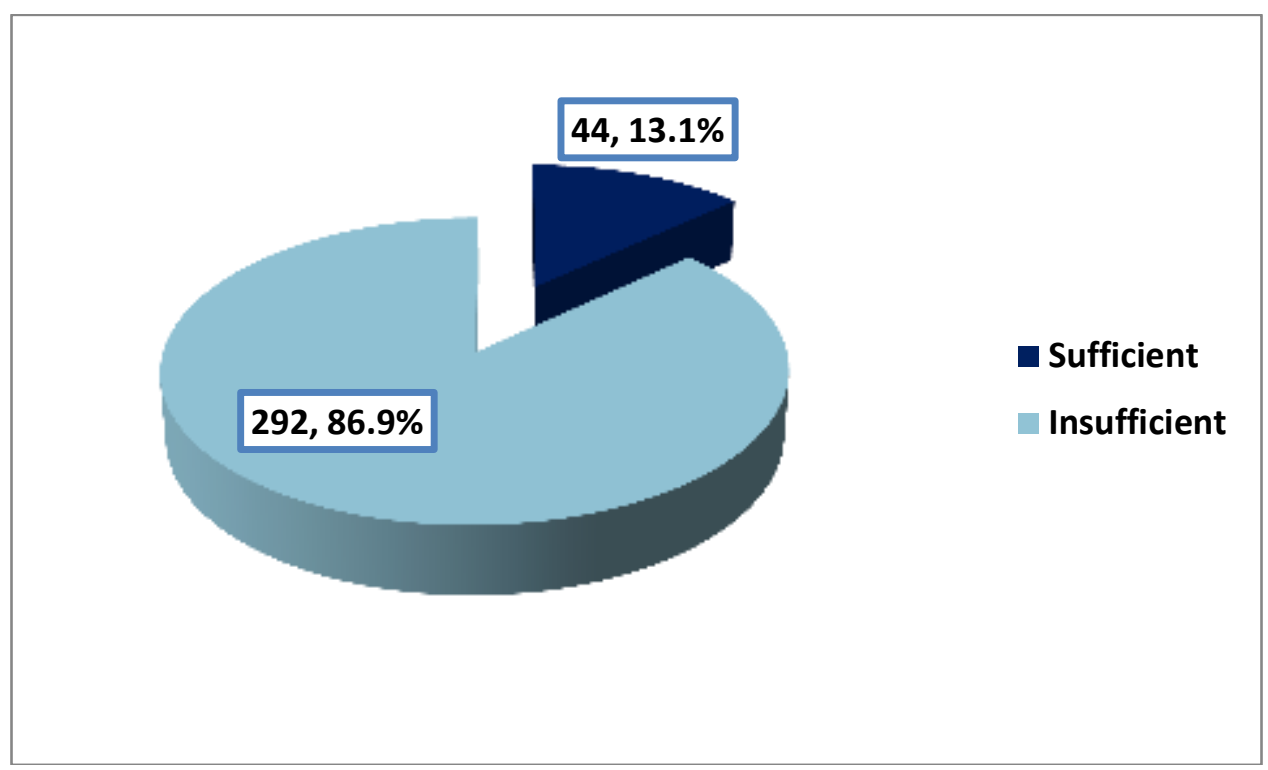

Figure 4:- Overall knowledge level of the participants regarding breast cancer.

\section{Discussion:-}

It has been evidenced that the occurrence of breast cancer in Arab world, including Saudi Arabia is about ten years younger than in European countries and USA. ${ }^{[35,36]}$ Also, it is well known that diagnosis of breast cancer at early stages would enable better survival. In Saudi Arabia, as in developing countries, breast cancer cases are presented at late stages and consequently with bad prognosis. ${ }^{[3]}$

Public awareness of breast cancer and its screening had significantly influence its early presentation. ${ }^{[9]}$ Therefore, this study was carried out to assess the knowledge and attitude about breast cancer among women in King Khalid Armed Forces Hospital, Tabuk.

Regarding knowledge of breast cancer symptoms, majority of the women (92.9\%) in the current study could recognize presence of a lump or thickness in breast, $47.3 \%$ recognized pain in one breast, $27.7 \%$ recognized that presence of a lump or thickness under armpit, changes in the size of breast or nipple (27.7\%) and discharge or bleeding from nipple (25.6\%) as symptoms of breast cancer. In a previous study carried out Qassim Region, Saudi 
Arabia, only $23 \%$ of the participants knew that breast cancer may present as breast lump, $18 \%$ recognized that it may present as pain in breast and $1.75 \%$ agreed that nipple discharge might be a symptom of breast cancer. ${ }^{[14]}$ In another more recent study carried out among University students in Al Madinah city, breast lump, pain, and nipple discharge were recognized by $55.3 \%, 58.7 \%$ and $65.3 \%$ of the students, respectively. ${ }^{[15]}$ In Oman, among women aged between 18 and 60 years, $19 \%$ had poor knowledge, $59 \%$ had average knowledge and $21 \%$ had good knowledge and only $1 \%$ had excellent knowledge about breast cancer symptoms. ${ }^{[16]}$ In Egypt, less than half of university students were aware of warning signs and symptoms of breast cancer. ${ }^{[1]}$ Worldwide; in United Kingdome, $18 \%$ of the women recognized five or more from non-lump symptoms of breast cancer. ${ }^{[18]}$ In Uganda, $61.3 \%$ of the participant women had intermediate level of knowledge about signs and symptoms of breast cancer. ${ }^{[19]}$

Regarding knowledge of breast cancer risk factors, 54.5\% of women in the current study could recognize past history of breast cancer whereas $36.3 \%$ and $27.7 \%$ of them knew that using hormonal replacement therapy and having a close relative with breast cancer are risk factor for breast cancer. In a study carried out by Alam in Riyadh (KSA), 30\% of the participants knew that use of oral contraceptives is a risk factor for breast cancer whereas $39.1 \%$ and $13.8 \%$ were aware of the facts that breast cancers may be hereditary and its risk increases with advancing age, respectively. ${ }^{[20]}$ In a study carried out among university students in Al-Madinah, ${ }^{[15]}$ hormonal therapy, hereditary and advanced age were recognized as risk factors for breast cancer by 36\%, 77.3\%, and 93.3\% of the students, respectively. Participants' knowledge about some risk factors for breast cancer such as overweight, having children later on in life or not at all, starting periods at an early age, having a late menopause, physical inactivity and aged of 50 years was very poor. This necessitates the need to educate them more about the risk factors associated with breast cancer.

Concerning breast cancer screening, minority of the women in the present study could recognize that the age at which women first invited to breast cancer screening programme according to guidelines of Saudi Ministry of Health is 50 years $(1.8 \%)$ and the age at which women receive last invitation to breast cancer screening programme according to guidelines of Saudi Ministry of Health is 69 years was recognized by only $0.3 \%$ of them. Also, in the present study, only $8 \%$ of the women reported that they were checking breast at least once per month whereas $15.5 \%$ checked them at least once every 6 months. Most of them $(76.5 \%)$ were not at all confident in noticing breast changes whereas only $1.2 \%$ of the participants were fairly confident. Almost one-quarter of them (27.4\%) saw a doctor about noticed breast changed whereas another $27.4 \%$ did not do this and $44.9 \%$ of the women never noticed breast changes. In a similar study carried out in UK, $52 \%$ women were confident about noticing the changes in their breast and $23 \%$ were checking their breast at least once per month and about $73 \%$ sought medical help within one week for noticing any breast changes. ${ }^{[18]}$ In a study carried out in Al-Madinah city among university students, $50.7 \%$ confirmed practice of breast self-examination (BSE). ${ }^{[15]}$ Kashgari and Ibrahim in a survey of Saudi women in Jeddah noted that only $12 \%$ of the respondents performed BSE. ${ }^{[21]}$ Similarly Ravichandran et al., reported from Riyadh region that only $23.1 \%$ of the subjects performed BSE. ${ }^{[22]}$ In Abha, $41.5 \%$ of women attending primary health care centers knew the breast self examination and only $29.7 \%$ performed breast self examination . ${ }^{[23]}$ In Malaysia, $25.5 \%$ of women aged over 20 years performed breast self-examination. ${ }^{[24]}$ In Nigeria, $70.7 \%$ of women reported that they didn't know how to perform breast self-examination and only $8.1 \%$ knew the exact time of performing breast self examination. ${ }^{[25]}$ In Bangladesh, only 8\% of women aged 30-59 years reported doing clinical breast examination. ${ }^{[26]}$ In Uganda, $76.5 \%$ of university students performed breast self-examination. ${ }^{[19]}$ In Yemen, $89 \%$ of women had never performed breast cancer screening. ${ }^{27]}$ In Egypt, only $8.8 \%$ of university students identified the time of performed breast self-examination and only $1.3 \%$ performed it regularly every month. ${ }^{[17]}$ In Gaza city, $75 \%$ of women had never undergone clinical breast examination, $24.6 \%$ were not knowing any screening methods and about half had misconception about breast screening. ${ }^{[28]}$ In Iraq, $30.3 \%$ of women aged between 18 and 55 years performed breast examination and $42 \%$ did not know how to perform breast self examination. ${ }^{[29]}$

The relatively low rate of practicing BSE in this study may be due to the female modesty which is considered a virtue in Islam. In addition, a cancer diagnosis is often associated with a stigma in KSA. ${ }^{[30]}$ So the women may not undergo breast cancer screening out of fear. Also, strict gender segregation is the social norm in KSA. Although religious guidelines allow Muslim women to take care of their health, and have their bodies examined by health care professionals for medical reasons, ${ }^{[31]}$ there is strong preference for a female health professional. ${ }^{[32,33]}$ The reason could be the objection raised by their husbands or male relatives to breast examination as males are considered the guardians of the females in middle east. ${ }^{[34]}$ Lastly, in KSA, the women are caretakers and tend to spend all their time focusing on the needs of family members before they focus on their own health needs. 
In the present study, the sufficient knowledge regarding breast cancer was observed among $13.1 \%$ of the participants, which is lower than that reported in another study carried out among University students in AlMadinah, ${ }^{[15]}$ where $70.9 \%$ of the participants had good knowledge regarding different aspects of breast cancer. Also, it is lower than that reported in Yemen where two third of women had poor knowledge about breast cancer. ${ }^{\text {[27] }}$ In Jordan, $51.8 \%$ of women were aware of breast cancer. ${ }^{[35]}$ In Iraq among 508 women between 18 and 55 years, 61.2 $\%$ had poor knowledge about cancer breast. ${ }^{[29]}$ In Kuwait, $43.1 \%$ of women had overall good knowledge about breast cancer symptom and risk factors. ${ }^{[36]}$ The clear difference between these studies and our study could be attributed to variation in the characteristics of the target population as well as cultural and social norms prevail in the Saudi Society.

Variables that might affect the level of knowledge as revealed by the Logistic regression analysis in the present study were women's age and education level. This has been documented in another study. ${ }^{[37]}$ Also, the role of high education in increasing awareness regarding breast cancer has been confirmed in a study carried out by Amin et al in Saudi Arabia. ${ }^{[38]}$

In the present work, checking of the breasts at least once per month was more reported by younger, higher educated women and those with higher income. The same has been reported by other local ${ }^{[23]}$ and international studies. ${ }^{[18]}$

This study has some few limitations including recruitment of women from one health care facility in Tabuk (King Khalid Armed Forces hospital), so the findings of the study cannot be generalized. The cross-sectional design of the study doesn't prove causality between independent variables and dependent one. Despite these limitations, studies like this can enhance the knowledge regarding breast cancer among women in our society

\section{Conclusion:-}

Results of the current study suggest that minority of adult women attending family medicine clinic and women health center, King Khalid Armed Forces hospital, Tabuk have sufficient knowledge of breast cancer symptoms, risk factors and screening. Younger and higher educated women were more knowledgeable than others in this regard.

Regarding breast cancer symptoms, with the exception of lump or thickness in breast, knowledge was suboptimal. Concerning risk factors, important risk factors were unknowledgeable to women such as early menarche, late menopause and nulliparity. Regarding screening, knowledge was frustrating.

Most of the women rarely or never checked their breast and only $8 \%$ did this at least once a month. Also, most of them were not at all confident in noticing breast changes and almost one-quarter of them saw a doctor about noticed breast changes.

\section{Recommendations:-}

Based on results of the present study, the following are recommended

1. There is an urgent need to enforce knowledge of women attending family medicine clinic and women health center, King Khalid Armed Forces hospital, Tabuk regarding various issues related to breast cancer, through health education in the clinic for breast cancer . particularly for older and lower educated women.

2. Using screens in waiting area for educational video about breast cancer .

3. Encourage women to check their breast and educated them about the correct method and appropriate time for that.

\section{References:-}

1. World Health Organization. [Online]. Available from: http:// www. who. int/cancer/detection/breastcancer/en/index1.html

2. CDC gov. [Online]. Available from: https://www.cdc .gov/ cancer/ international/statistics.htm

3. Chs gov sa. [Online]. Available from: http://www.chs.gov.sa/ En/ HealthRecords/CancerRegistry/Pages/CancerRegistryRecords.aspx

4. Cancer Research UK. [Online]. Available from: http://www. cancerresearchuk.org/health-professional/cancerstatistics/statistics-by-cancer-type/breast-cancer/risk-factors

5. Cancer research UK org. [Online]. Available from: http://www. cancerresearchuk.org/about-cancer/type/breastcancer/about/breast-cancer-symptoms 
6. Mohgovsa.[Online]. Available from: http://www.moh.gov.sa/ endepts/TCP/ Documents/88. Breast Cancer - Use of Screening Strategies for the Detection of Breast Cancer.pdf

7. US preventive services task force org. [Online]. Available from: https://www.uspreventiveservicestaskforce.org/Page/Document/UpdateSummaryFinal/breast-cancer-screening1

8. Forbes LJ, Linsell L, Atkins L, Burgess C, Tucker L, Omar L, et al. A promoting early presentation intervention increases breast cancer awareness in older women after 2 years: a randomised controlled trial. $\mathrm{Br} \mathrm{J}$ Cancer. 2011;105:18-21.

9. Stapleton JM, Mullan PB, Dey S, Hablas A, Gaafar R, Seifeldin IA, et al. Patient-mediated factors predicting early- and late-stage presentation of breast cancer in Egypt. Psychooncology. 2011;20:532-537.

10. Jemal A, Bray F, Melissa MC, Jacques F, Elizabeth W, Forman D. Global cancer statistics. CA Cancer J Clin. 2011;61:69-90

11. Ramirez AJ, WestcombeAM, Burgess CC, Sutton S, Littlejohns P, Richards MA. Factors predicting delayed presentation of symptomatic breast cancer: a systematic review. Lancet. 1999;353:1127-1131

12. Al-Khamis NK. Low awareness of breast cancer and considerable Bbarriers to early presentation among Saudi women at a primary care setting. J Cancer Educ. 2016 Oct 10. [Epub ahead of print]

13. Cancer research UK org. Cancer Research UK. [Online]. Available from: http://www.cancerresearchuk.org/health-professional/prevention-and-awareness/the-cancer-awarenessmeasures-cam [Accessed 6 January 2017].

14. Jahan S, Al Saigul AM, Abdelgadir MH. Breast cancer knowledge, attitudes and practices of breast self examination among women in Qassim region of Saudi Arabia. Saudi Med J 2006; 27: 1737e1741.

15. Latif R. Knowledge and attitude of Saudi female students towards breast cancer: A cross-sectional study. Journal of Taibah University Medical Sciences 2014;9(4): 328e334

16. Renganathan L, Ramasubramaniam S, Al-Touby S, Seshan V, Al-Balushi A, Al-Amri W, et al. What do Omani Women know about Breast Cancer Symptoms? Oman Med J. 2014 Nov;29(6):408-13.

17. Boulos DNK, Ghali RR Awareness of breast cancer among female students at Ain Shams University, Egypt. Glob J Health Sci. 2014 Jan; 6(1): 154-161.

18. Forbes LJ, Atkins L, Thurnham A, Layburn J, Haste F, Ramirez AJ. Breast cancer awareness and barriers to symptomatic presentation among women from different ethnic groups in East London. Br J Cancer. 2011 Nov 8;105(10):1474-9.

19. Godfrey K, Agatha T, Nankumbi J. Breast cancer knowledge and breast self-examination practices among female University students in Kampala, Uganda: A descriptive study. Oman Med J. 2016 Mar; 31(2): 129-134

20. Alam AA. Knowledge of breast cancer and its risk and protective factors among women in Riyadh. Ann Saudi Med 2006; 26: $272 \mathrm{e} 277$.

21. Kashgari RH, Ibrahim AM. Breast cancer: attitude, knowledge and practice of breast self examination of 157 Saudi women. J Family Community Med 1996; 3: 10e13.

22. Ravichandran K, Al-Hamdan NA, Mohamed G. Knowledge, attitude and behavior among Saudis toward cancer preventive practice. J Family Community Med 2011; 18: 135e142.

23. Mahfouz AA, Hassanein MH, Nahar S, Farheen A, Gaballah II, Mohamed A, et al. Breast cancer knowledge and related behaviors among women in Abha City, southwestern Saudi Arabia. J Cancer Educ. 2013 Sep;28(3):516-20.

24. Akhtari-Zavare M, Juni MH, Ismail IZ, Said SM, Latiff LA. Barriers to breast self examination practice among Malaysian female students: a cross sectional study. Springerplus. 2015 Nov 11;4:692

25. Oladimeji KE, Tsoka-Gwegweni JM, Igbodekwe FC, Twomey M, Akolo C, Balarabe HS, et al. Knowledge and beliefs of breast self-examination and breast cancer among market women in Ibadan, South West, Nigeria. PLoS One. 2015 Nov 25;10(11):e0140904.

26. Islam RM, Bell RJ, Billah B, Hossain MB, Davis SR. Awareness of breast cancer and barriers to breast screening uptake in Bangladesh: A population based survey. Maturitas $2016 \mathrm{Feb} ; 84: 68-74$.

27. Sakkaf KA, Basaleem HO. Breast cancer knowledge, perception and breast self- examination practices among Yemeni women: an application of the health belief model. Asian Pac J Cancer Prev. 2016;17(3):1463-7.

28. Abu-Shammala BI, Abed Y. Breast cancer knowledge and screening behavior among female school teachers in Gaza City. Asian Pac J Cancer Prev. 2015;16(17):7707-11.

29. sHasan TN, Shah SA, Hassan MR, Safian N, Azhar ZI, Syed Abdul Rahim SS, et al. Poor knowledge and practice towards breast cancer among women in Baghdad City, Iraq. Asian Pac J Cancer Prev. 2015;16(15):6669-72.

30. Bener A, Alwash R, Miller CJ, Denic S, Dunn EV. Knowledge, attitudes, and practices related to breast cancer screening: a survey of Arabic women. J Cancer Educ 2001; 16: 215e220. 
31. Al-Khayat. Right path to health: health as a human right in Islam. WHO Eastern Mediterranean Regional Office; http:// applications.emro. who.int/ dsaf/ dsa217.pdfwebcite.

32. Ayash C, Axelrod D, Nejmeh-Khoury S, Aziz A, Yusr A, Gany FM. A community intervention: AMBER: Arab American breast cancer education and referral program. J Immigr Minor Health 2011; 13: 1041e1047.

33. Bou Khalil R. Attitudes, beliefs and perceptions regarding truth disclosure of cancer-related information in the Middle East: a review. Palliat Support Care 2013; 11: 69e78.

34. Donnelly TT, Al Khater AH, Al-Bader SB, Al Kuwari MG, AlMeer N, Malik M, et al. Beliefs and attitudes about breast cancer and screening practices among Arab women living in Qatar: a cross-sectional study. BMC Women's Health 2013; 13: 49.

35. Suleiman AK. Awareness and attitudes regarding breast cancer and breast self-examination among female Jordanian students. J Basic Clin Pharm. 2014 Jun;5(3):74-8.

36. Saeed RS, Bakir YY, Ali LM. Are women in Kuwait aware of breast cancer and its diagnostic procedures? Asian Pac J Cancer Prev. 2014; 15(15):6307-13.

37. Ibrahim EM, Al-Idrissi HY, Al-Khadra AH, Kurashi NY, AlJishi FM, Saied I, et al. Women's knowledge of and attitude toward breast cancer in a developing country: implications for program intervention results based on interviewing 500 women in Saudi Arabia. J Cancer Educ 1991; 6(2): 73e81.

38. Amin TT, Al Mulhim AR, Al Meqihwi A. Breast cancer knowledge, risk factors and screening among adult Saudi women in a primary health care setting. Asian Pac J Cancer Prev. 2009 Jan-Mar;10(1):133-8 